tree-level 2; and 'cooking methods' such as boiling, frying, formed tree-level 3. The associations between particular food intakes and health outcomes (presence of NAFLD and significant fibrosis, diabetes mellitus, hypertension, dyslipidaemia, and cardiovascular events) were determined by logistic regression.

Results Individuals with NAFLD consumed significantly higher amount of cereals (as refined rice), fat and edible oils (as animal fat), meat (as red meat), sugar (as refined sugar) and fried foods, and lower amount of vegetables, pulses, nuts, seeds, and milk compared to controls (all $\mathrm{P}<0.05$ ). Consumption of meat (as red meat), fats (as animal fat), nuts and refined rice was positively associated with both the presence of NAFLD and its severity (significant fibrosis), whereas, consumption of vegetables (as leafy vegetables), fruits, oily seeds, spices, and dried pulses was negatively associated with NAFLD. Fried and boiled food consumption were positively and negatively associated with NAFLD, respectively. Increased consumption of animal fats was associated with diabetes, hypertension, and cardiovascular outcomes, whereas consumption of wholegrain rice was negatively associated with these health-related outcomes.

Conclusions Comprehensive evaluation of food intakes using validated FFQ and tree-based approach in a large, well-characterised population-based cases and controls has enabled the identification of specific dietary indicators associated with NAFLD, its severity and the co-morbidity cluster. These findings provide a basis for culturally sensitive advice to prevent the development of NAFLD as well as the design of individualised intervention in those with NAFLD.

\section{A DOUBLE BLIND, RANDOMISED, PLACEBO- CONTROLLED STUDY TO ASSESS SAFETY AND TOLERABILITY OF ORAL ENTEROSORBENT CARBALIVE (YAQ-001) IN CIRRHOTIC PATIENTS}

${ }^{1} J a n e$ Macnaughtan*, ${ }^{2}$ Agustin Albillos, ${ }^{1}$ Annarein Kerbert, ${ }^{3}$ Victor Vargas, ${ }^{4}$ Francois Durand, ${ }^{5}$ Pere Gines, ${ }^{5}$ Elsa Sola, ${ }^{6}$ Lindsey Edwards, ${ }^{1}$ Simon Eaton, ${ }^{7}$ Jane Cox, ${ }^{1}$ Fausto Andreola, ${ }^{1}$ Amir Gander, ${ }^{2}$ Javier Martinez, ${ }^{8}$ Giacomo Zaccherini, ${ }^{1}$ Gautam Mehta, ${ }^{3}$ César Jimenez, ${ }^{9}$ Kathrin Husi, ${ }^{10}$ Christopher Stabile-Barnett, ${ }^{2}$ Miguel Rodriguez-Gandia, ${ }^{11}$ Mar Hernandez Obiols, ${ }^{11}$ Roberto Elosua, ${ }^{11}$ Joan Villa, ${ }^{12}$ Alicia Navarro, ${ }^{13}$ Carrie Morgan, ${ }^{13}$ Karen Church, ${ }^{13}$ Michal Kowalski, ${ }^{13}$ Edouard Guillabert, ${ }^{13}$ Katherine Avery, ${ }^{13}$ Tom Avery, ${ }^{13}$ Daniel Green, ${ }^{13}$ Lynda McConaghy, ${ }^{14}$ Susi Sandeman, ${ }^{1}$ Nathan Davies, ${ }^{15}$ Marco Pavesi, ${ }^{1}$ Raj Mookerjee, ${ }^{15}$ Vicento Arroyo, ${ }^{16} \mathrm{Helena}$ Cortez-Pinto, ${ }^{9}$ Reiner Wiest, ${ }^{8}$ Paolo Caraceni, ${ }^{1}$ Rajiv Jalan. ${ }^{1}$ University College London, UK; ${ }^{2}$ Hospital Ramón y Cajal, Madrid, Spain; ${ }^{3}$ Hospital Vall d'Hebron, Barcelona, Spain; ${ }^{4}$ Hospital Beaujon, Clichy, France; ${ }^{5}$ Hospital Clinic, Barcelona, Spain; ${ }^{6}$ King's College London, UK; ${ }^{7}$ Institute of Hepatology, London, UKi ${ }^{8}$ University of Bologna, Italy; ${ }^{9}$ University Hospital Bern, Switzerland; ${ }^{10}$ A2F-Associates Ltd, UK; ${ }^{11}$ Datarus, Barcelona, Spain; ${ }^{12}$ AlphaBioresearch, Madrid, Spain; ${ }^{13}$ Yaqrit Ltd., UKi ${ }^{14}$ University of Brighton, UK; ${ }^{15}$ EF-CLIF, Barcelona, Spain; ${ }^{16}$ Universidade de Lisboa, London, Portugual

\subsection{6/gutjnl-2021-BASL.9}

Background Yaq-001 is a non-absorbable, synthetic carbon with high adsorptive capacity for bacterial products including lipopolysaccharide (LPS) and pro-inflammatory cytokines. Studies in cirrhotic rats showed reduced endotoxemia, improvements in end-organ dysfunction and reduced sensitisation to LPS. The aims of this study were to evaluate the safety and tolerability of Yaq-001 in decompensated cirrhotic (DC) patients and characterise immunological and metabolic effects. Methods This EU-funded H2020 study (CARBALIVE:634579) recruited patients with diuretic-responsive cirrhotic ascites. 28-

Abstract 009 Figure 1 Effect of Yaq-001 treatment on indices of systemic (A) and gut (B) inflammation and (C) gut permeability (SD+SEM). HC and Decomp values from PMID: 32838247 
patients from 8-EU centres were randomised to receive $4 \mathrm{~g}$ of Yaq-001 (Y) or placebo (P) for 12-weeks. Safety assessments were performed at baseline, weeks 1, 4, 8 and 12. Endotoxin activity assay (EAA, data used for analysis: CV $<20 \%$ ), organ function, nutrition, markers of systemic and gut inflammation, gut permeability, urinary 1HMRS, and next generation sequencing of stool were evaluated at baseline, W4 and W12. Data were summarized by group and visit as means (SD) with select efficacy parameters modeled using MMRM.

Results 14-patients were randomised to each group (Y: Age: 57.8 \pm 7 yrs, M: 71\%, MELD: 12.4 \pm 0.9 ; P: Age: 57.3 \pm 10 ,

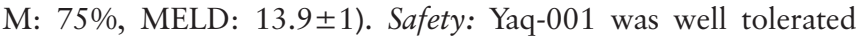
with no SAEs, stable MELD score, decompensation events and nutritional status. Compliance: 93\%(Y) 67\%(P). Endotoxemia: Trends to reduction in EAA ratio (Y:-2.4\% vs P:29.6\%) and constitutive whole blood ROS (Y:-37.3\% vs P:+34.3\%) were observed in the active group (figure 1). Systemic inflammation: Reductions in WCC, CRP, IL6 and CXCL10 in the active group were observed compared with placebo $(\mathrm{Y}(\%)$ :8.8,-4.3,-3.6,-16.7; $\mathrm{P}(\%)+0.4,+23.9,+13.8,+31.8$ ) (figure 1). Gut inflammation/permeability: Improvements in faecal cytokines in Yaq-001 patients trended towards published values for healthy control. Reduction in plasma D-lactate was observed in Yaq-001 compared to placebo suggestive of an improvement in gut barrier integrity (figure 1). Metabolism: Yaq-001 reduced stool ammonia with a trend to increased urinary hippurate. Microbiome: No change in bacterial diversity was observed.

Conclusion Yaq-001 was safe and well tolerated. The data suggest proof of mechanism that Yaq-001, modulates systemic endotoxemia and inflammation by impacting gut inflammation and its permeability.

\section{GRANULOCYTE-COLONY STIMULATING FACTOR (G-CSF) TO TREAT ACUTE-ON-CHRONIC LIVER FAILURE; RESULTS OF THE FIRST MULTICENTER RANDOMIZED TRIAL (GRAFT STUDY)}

Cornelius Engelmann*, Adam Herber, Tony Ildh, Ingolf Schiefke, Alexander Zipprich, Anett Schmiedeknecht, Stefan Zeuzem, Tobias Goeser, Ali Canbay, Christoph Berg, Jonel Trebicka, Frank E Uschner, Tobias Mueller, Niklas Aehling, Moritz Schmelzle, Katrin Splith, Frank Lammert, Christian M Lange, Christoph Sarrazin, Christian Trautwein, Michael Manns, Dieter Häussinger, Jan Pfeiffenberger, Peter Galle, Annegret Franke, Thomas Berg. Ildh - University College London; Charite - Universitaetsmedizin Berlin, Germany

\subsection{6/gutjnl-2021-BASL.10}

Background The acute-on-chronic liver failure (ACLF) is a severe complication of cirrhosis characterised by immune dysfunction and high mortality. Granulocyte-colony stimulating factor (G-CSF) mobilises immunomodulatory stem and immune cells and has been proposed as therapy for ACLF. We therefore conducted the first multicentre randomised trial. Methods In this prospective, controlled, open-label 2-armed study 176 patients with ACLF according to the EASL-CLIF criteria without malignancies or septic shock were randomised in 18 trial centres between $02 / 2016$ and $01 / 2019$ in a $1: 1$ ratio to receive either standard medical therapy (SMT) or SMT plus G-CSF (12 injections with $5 \mu \mathrm{g} / \mathrm{kg}$ for 5 days daily, then every 3 days). The primary efficacy endpoint was 90-day transplant free survival (TFS90).
Results The 90-day transplant free survival was 34.1\% in the G-CSF plus SMT group compared to $37.5 \%$ in the SMT group with a corresponding hazard ratio (HR) of $1.05(95 \%$ CI $0.711 ; 1.551)(p=0.805)$. G.CSF had no effect on the 360-day transplant free survival [HR of 0.998 [95\%CI $0.697 ; 1.430(\mathrm{p}=0.992)$ ] or overall survival [HR of 1.058 [95\%CI $0.727 ; 1.548(\mathrm{p}=0.768)]$. There was no improvement of the CLIF-C OF score $(p=0.757)$, MELD score $(p=0.884)$ or the rate of bacterial infections $(p=0.251)$ with G-CSF therapy. In subgroups of patients without infections $[p=0.883]$, with alcohol related ACLF $[p=0.875]$, or with ACLF defined by the APASL criteria $[p=0.405]$ G-CSF also didn't improve survival. There were 61 serious adverse events (SAE) reported in the G-CSF+SMT group and 57 SAEs in the SMT group including seven drug-related serious adverse reactions under G-CSF therapy. The study was prematurely terminated due to futility.

Conclusion This interim analysis of the first multicentre trial reveals that G-CSF has no therapeutic effect in ACLF, which is in obvious contrast to the results from smaller clinical trials published previously. As a consequence, the study was prematurely terminated due to futility after conditional power calculation.

\section{P001 RITUXIMAB INITIATION, PRESCRIBING AND HEPATITIS B REACTIVATION: RETROSPECTIVE FIVE-YEAR REVIEW}

Zillah Cargill ${ }^{*}$ Nicola Grasso, George Bird. Maidstone Hospital, Maidstone and Tunbridge NHS Trust, UK

\subsection{6/gutjnl-2021-BASL.11}

Introduction Hepatitis B reactivation is a potentially life-threatening complication of immunosuppression in patients with serological evidence of current or past exposure to Hepatitis B Virus (HBV). Rituximab is a widely used B-cell depleting medication widely used to treat haematological cancers and rheumatological disease. The British Society of Rheumatology, British Society of Haematology and the European Association of Study of the Liver guidelines all recommend patients must all be assessed for HBV before rituximab initiation. This is a single-centre five-year retrospective review of our Trusts Rituximab prescribing and HBV screening.

Methods We included all adult patients receiving rituximab between 1st October 2015 and 30th September 2020 at our Trust. Patients were identified using hospital pharmacy Rituximab prescribing logs. Indications, HBV screening bloods and outcomes were collected using electronic records (Allscripts, Telepath). Patients with incomplete or missing records were excluded.

Results A total of 870 patients were included in this study. Seventy percent $(\mathrm{n}=611)$ were for oncohaematological indications and $30 \%(\mathrm{n}=258)$ for rheumatological. There were 606 patients newly initiated on Rituximab during the study period (table 1). HBV screening improved from $62 \%$ to $94 \%$ during the study period.

Eighteen patients were positive or equivocal for Hepatitis B core antibody and 1 for Hepatitis B Surface Antigen. Four patients were prescribed lamivudine prophylaxis, 2 Tenofovir and 1 entecavir. The other patients had no treatment or monitoring. Only 1 case was on adequate length of 